\title{
Urban Health Education: Global Challenges and Opportunities
}

\author{
Jason Corburn $(\mathbb{D} \cdot$ Blaise Nguendo-Yongsi • \\ Waleska Teixeira Caiaffa • Tolu Oni • Gerard Salem
}

Published online: 31 May 2019

(C) The New York Academy of Medicine 2019

On a planet of cities, a new cadre of researchers and professionals are needed to understand and address the human health challenges of the twenty-first century

\section{J. Corburn $(\bowtie)$ \\ School of Public Health \& Department of City and Regional Planning, University of California, Berkeley, CA, USA \\ e-mail: jcorburn@berkeley.edu}

\section{B. Nguendo-Yongsi}

Institute for Training \& Research in Population Studies (IFORD), The University of Yaoundé II, Soa, Cameroon

e-mail: sir_nguendoyongsi@hotmail.com

\section{W. T. Caiaffa}

School of Medicine, Federal University of Minas Gerais, Belo Horizonte, Brazil

e-mail: caiaffa.waleska@gmail.com

\section{T. Oni}

Medical Research Council Epidemiology Unit, University of Cambridge, Cambridge, UK

e-mail: Tolullah.oni@mrc-epid.cam.ac.uk

\section{T. Oni}

Division of Public Health Medicine, School of Public Health and Family Medicine, University of Cape Town, Cape Town, South Africa

T. Oni

Stellenbosch Institute for Advanced Study, Stellenbosch, South Africa

G. Salem

Department of Geography, Université Paris-Nanterre, Nanterre, France

e-mail: salem.gerard@gmail.com urbanization. How to prepare these action-researchers, decision makers, activists, and engaged citizens is the question addressed in this commentary.

The Sustainable Development Goals, the New Urban Agenda, and reports from the Intergovernmental Panel on Climate Change in 2018 have all made clear that the health of the planet and populations will depend on whether and how we improve well-being in cities. More people than ever before are living in cities, and this shapes the environmental, economic, political, and health status for all. How can we prepare this and future generations to ensure that urban life is healthy, equitable, and sustainable for all? We suggest that in order to address the twenty-first century challenge of urban health, new education and training in universities and professional settings is needed. To explore this issue, an Urban Health Education working group was established by the International Society of Urban Health (ISUH) in 2015. The authors here are all members of that working group, and we have met over the past 3 years to frame the challenges of urban health education, explore ways to build-upon existing university training programs in urban health, and define principles and ideas for articulating the competencies for the twenty-first century urban health practitioner. The group held three global workshops in San Francisco, Coimbra, and Kampala, with educators and practitioners from universities that cover North and South America, Europe, Eastern and Southern Africa, the Middle East, Asia, and Australia. The ideas shared here reflect some of the themes that have emerged from this working group and are intended to share our work with a broader audience and act as an invitation to other scholars, practitioners, and activists to join our on-going efforts. 


\section{Urban Health Education: a New Discipline}

What exactly is urban health and why do we need new training? In 2003, Vlahov and Galea published "Urban Health: A New Discipline" in the Lancet [1], where they identified some key features of the field relevant for our discussion of urban health education. First, urban health research is the domain of multiple disciplines, including but not limited to epidemiology, biology, sociology, planning, geography, political science, architecture, and engineering. Thus, urban health is a field that is concerned "with the determinants of health and diseases in urban areas and with the urban context itself as the exposure of interest. As such, defining the evidence and research direction for urban health requires that researchers and public health professionals pay attention to theories and mechanisms that may explain how the urban context may affect health and to methods that can better illustrate the relation between the urban context and health" [2].

Second, these disparate academic disciplines rarely work together with practitioners to collaboratively study and act upon the factors in urban areas that influence health. We note a third related feature from our work: few universities have developed training programs that prepare scholars and practitioners with the interdisciplinary skills likely needed to understand and improve urban health, yet our students are increasingly demanding new approaches to move from understanding to urban action. Based on our workshops with educators from around the world, we have identified some challenges for building an educated workforce engaged with the challenges of global urban health. These challenges include:

1) There are few university-level training programs in urban health, whether at the undergraduate or postgraduate level. The few that exist tend to be located in schools of public health, urban planning, geography, or environmental science/studies.

2) An increasing number of universities offer at least one class/course in urban health, but these tend to focus either on a set of specific diseases in cities (i.e., either NCDs or communicable, but rarely both) or physical, built, social, or health care environments.

3) The limited university education programs in urban health focus on a narrow set of methodological training, such as spatial epidemiology, and/or neighborhood ethnography, but few also offer training in mixed methods, participatory action research, or policy analysis.

4) Urban health education in schools of public health does not expose students to understanding urban governance, or the institutions, social movements, economic policies, laws, and regulations that combine to shape who is included in decisions and the transparency of decision-making.

5) A limited number of university programs offer mentored urban health practicum or field-based learning opportunities. Exceptions were the clientoriented studio-model used in architecture and urban planning.

6) Few urban health education programs delve deeply into one or more cities or compare across cities, preferring to frame the issue generally, and there are few university-based urban health training programs focused on urbanizing regions in subSaharan Africa, Asia, or Latin America, where rapid urbanization is taking place.

\section{Toward a New Model of Urban Health Education}

Drawing from input to the ISUH working group and our own experiences teaching urban health, we offer the following "call to action" for a new approach to urban health education based on five core themes; history, place, equity, fieldwork, and urban science. We hope to inform existing university degree programs and perhaps stimulate the creation of new, interdisciplinary degrees in urban health.

1) Urban health history. All urban health education must begin with a critical review of the histories of public health, medicine, urbanization, development, colonialism, and related ideas. Urban health practitioners must be fluent with national and global trends, processes, and policies that shape the wellbeing of urban populations today. We cannot understand how to address urban health today, and in the future, if we do not trace the origins of the factors shaping urban development, population health and risk that disproportionately burdened some urban populations and places. As George Rosen, former editor of the American Journal of Public Health and author of "Health, History and the Social Sciences," noted: 
Every social phenomenon is the result of historical process, that is societal factors operating over a period of time through human interaction...As soon as large-scale phenomena are investigated, account must be taken of the historical facet. This applies as well to the results (theories, models, data et alia) of researches [3]. (p. 55).

Further, we suggest that this history must be read from the perspectives of those who lived it, not just those analyzing it from afar, especially to understand the legacies of the brutal experiences of slavery, colonialism, eugenics, racism, segregation, sexism, and other ideas that continue to shape urban health science and policy today.

2) Place and urban health. While urban health training tends to focus on "built environments" or "neighborhood effects" and health, this approach misses how geographic space becomes a "place"and thus can influence health. Studying place requires going beyond measures of static variables in space, but rather understanding the ways place is created and re-created by institutions and people through the assigning of economic, social and political value. Urban placemaking is fundamentally about governance, and so education must engage with the norms, laws, politics, and social movements of cities and municipal regions. A critical engagement with place can ensure urban health scholars understand some of the key dynamics of urbanization, such as displacement, diaspora, and migration.

3) Health equity and well-being. Today's cities are too often characterized by stark inequality, between the wealthy and poor and different racial/ethnic groups, among other differences. Urban informal settlements are some of the starkest manifestation of the failure of urbanization to create equitable and healthy living conditions [4]. Epidemiologists and others continue to measure population differences in health status, and urban health education will need to link these analyses to emerging methods that capture multiple and cumulative exposures in urban settings. Urban health equity is also ensuring just and fair inclusion of all people in a city while addressing and eliminating the systems and institutional barriers that prevent some groups from being healthy. Equity often raises challenges of contested values, so practitioners must be skilled in understanding and incorporating different cultural and other belief systems into research and practice. In some instances, multiple adverse insults in urban settings, from poverty to discrimination to violence, can act as "toxic stressors" that alter human biology and contribute to infection susceptibility, mental illness, and some chronic diseases. Urban health equity demands that training is equally focused on identifying and analyzing the relative influences of urban health assets, or the positive aspects of urban life that can promote health and well-being for all. Equity also demands training in how to work with, not on, communities, and this will surely differ from city to city.

4) Field Sites. There is a long tradition in public health of including field- and client-based learning as part of education and professional training [5]. These field sites can range from neighborhood clinics and legal services to client-based urban design and architecture studios. Urban health might re-engage with the ideas of "schools without walls" oriented toward cities and community participation. Education in partnership building, collaboration, negotiation, conflict resolution, participatory policy-making, and formative evaluation are necessary for field-site work, but so too will be understanding local health care systems. This will ensure that universities train their urban health students in how to engage locally, while recognizing how "local" economies and culture are also influenced by global dynamics. We suggest that global urban health can be understood by learning about a multiplicity of places, rather than moving toward universal theories that can wash-out local culture, people, and institutions. Yet, urban health education must also avoid an extraction model of field-based education - where information and data only flow out of communities to researchers-to more sustained urban health learning that contributes to meaningful knowledge and skill-building with urban communities. We emphasize a learning-bydoing approach here, such as community-based participatory research (CBPR) and citizen science.

5) New Urban Science. What all the above (history, place, equity, field sites) suggest to us is the need for a new urban health science. As Cuto et al. note: "Discrete research communities, ranging from hydrology, health sciences, criminology, or finance, are unable to jointly advise city planners or 
community leaders on the complex multidimensional nature of urban problems - or indeed on the most appropriate prioritization of urban solutions." [6] We agree that this new urban health science is not just about bringing disparate disciplines together, but will raise fundamental question about what is legitimate evidence and who is "expert" enough to co-produce knowledge for urban health? Further, the complexity of urban systems will likely demand an adaptive management approach, where decisions are made under conditions of technical and social uncertainty and must include robust systems of monitoring and learning to adjust as new information emerges.

\section{Urban Health Education: Moving Forward Together}

What we have proposed here are some ideas for the twenty-first century global urban health education. We are calling for universities to re-engage locally-with their community, city, or region - and to also build a global network of urban health educators to promote comparative urban health analyses. Urban inequalities and the rapid pace of change suggest we cannot wait for traditional evidence-to-action paradigms or continue to train students in the same way and expect different outcomes. In fact, our students are demanding change to our institutions, and we aim to be responsive to them and the global challenges we face for population health and well-being. Critical historical analyses, participatory methods, action-research, and adaptive strategies should frame education for the new urban health science. In order to operationalize urban health education, schools of medicine, public health, nursing, and others will need to collaborate with the social sciences, law, education, planning, and architecture. We hope this commentary contributes to a much needed global conversation about the current state and future of urban health education.

Acknowledgements The authors would like to thank the participants in the ISUH Education \& Training Workshops for valuable input.

Funding The authors received no financial support for the research, authorship, and/or publication of this article.

\section{References}

1. Vlahov D, Galea S. Urban health: a new discipline. Lancet. 2003;362:1091-2.

2. Galea S, Vlahov D. Urban health: evidence, challenges, and directions. Annu Rev Public Health. 2005;26:341-65.

3. Rosen G. Health, History and the social sciences. In, George Rosen, from medical police to social medicine: essays on the history of health care. New York, NY: Science History Publications; 1973.

4. Corburn J, Sverdlik A. Slum upgrading and health equity. IJERPH. 2017;14:342. https://doi.org/10.3390 /ijerph14040342.

5. Fee E, Bu L. Models of public health education: choices for the future? Bull World Health Organ. 2007;85(12):977-9.

6. Cuto M, Parnell S, Seto KC. Building a global urban science. Nat Sustain. 2018;1:2-4. https://doi.org/10.1038/s41893017-0013-9.

Publisher's Note Springer Nature remains neutral with regard to jurisdictional claims in published maps and institutional affiliations. 